\title{
SENSITIVITY AND SCENARIO ANALYSIS FOR SIMULATION METAMODELS
}

\author{
Susan M. Sanchez \\ L. Douglas Smith \\ Edward C. Lawrence \\ School of Business Administration \\ University of Missouri-St. Louis \\ 8001 Natural Bridge Road \\ St. Louis, Missouri 63121-4499, U.S.A.
}

\begin{abstract}
We use simple orthogonal and non-orthogonal designs to analyze a multi-tiered model for forecasting performance of a large-scale home mortgage portfolio. The experiments are used to assess the sensitivity of performance to projected changes in economic conditions, as well as the sensitivity of the model to coefficients estimated from historical data. Our results attribute the variation in loan performance to variation in individual factors or factor combinations, indicating which are crucial to monitor or forecast accurately. The results are at times counter-intuitive, indicating the benefits of a systematic approach to sensitivity assessment and scenario generation.
\end{abstract}

\section{INTRODUCTION}

Managing credit risk in financial institutions requires the ability to forecast aggregate losses on existing loans, to predict the length of time that loans will be on the books before prepayment or default, to analyze the expected performance of particular segments in the existing portfolio, and to project payment patterns of new loans. To accomplish these tasks, Smith et al. (1996) developed a comprehensive forecasting model in SAS for a major California financial institution. The comprehensive model consists of three components, as summarized in Table 1. The first component is a model of transition probabilities among five financial states. Current loans are those with outstanding balances and payments on schedule, delinquent loans ( 30 to 89 days or $90+$ days) have outstanding balances and payments overdue, paid-off loans have had the outstanding balance paid in full at maturity or earlier, and defaulted loans are those for which partial or complete charge-off has occurred and/or the title has been acquired through foreclosure.

The transition component of the comprehensive model is structured as a Markovian recursion to predict the likelihoods that a loan will be in each alternative state at annual intervals. In aggregate, it predicts the expected number of accounts in the portfolio that will fall in each financial state. At the beginning of the forecast period, the state of an active account will either be current, delinquent 30 to 89 days, or delinquent $90+$ days. Each time a payment is due, the debtor may make a payment to remain current, delay payment, or prepay the loan to maturity. Over the scheduled term of the loan, it is possible that the loan can be in any of the five financial states. The loan will either be in the paid-off state or defaulted state after maturity. Let

$$
\begin{aligned}
P_{j}(t)= & \operatorname{Pr}\{\text { loan is in state } j \text { at time } t\} \\
P_{k, j}(t)= & \operatorname{Pr}\{\text { loan is in state } j \text { at time } t+1\} \\
& \text { given it is in state } k \text { at time } t\}
\end{aligned}
$$

Allowing for non-stationary transitional probabilities, the likelihood that a loan is in state $j$ at time $t+1$ is expressed as a Markov chain as follows:

$$
P_{j}(t+1)=\sum_{k=1}^{5} P_{k}(t) \times P_{k, j}(t) .
$$

Transitions can be made from the three active states (current, delinquent 30-89 days, or delinquent $90+$ days) to each of the other five states. However, the defaulted and paid-off states are absorbing (terminal) states from which no transitions can be made.

The second component models the likelihood of a loss occurring after transition to the defaulted state. The magnitude of the expected loss depends on the amount of equity in the property and the position of the lien holder. The net equity is affected by changes in market price of the property and by the extent to which the principal has been paid off. Because there is no loss on a substantial portion of loans which terminate with default, and because loss patterns may 
Table 1: Structure of the Comprehensive Forecasting Model

\begin{tabular}{|c|c|c|c|}
\hline & \multicolumn{3}{|c|}{ Component } \\
\hline & Transition & Loss & Severity \\
\hline Description & $\begin{array}{l}\text { Yearly transition probabilities } \\
\text { from the three active states to } \\
\text { the five potential states }\end{array}$ & $\begin{array}{l}\text { Probabilities of defaulted loan } \\
\text { transitions into four potential } \\
\text { states }\end{array}$ & $\begin{array}{l}\text { Proportion of outstand- } \\
\text { ing balance charged off }\end{array}$ \\
\hline Model & 1. Current & ORE/Loss incurred & Total loss \\
\hline States & 2. Delinquent $30-89$ days & ORE/No loss incurred & Partial loss \\
\hline & 3. Delinquent $90+$ days & Non-ORE/Loss incurred & \\
\hline & 4. Paid-off & Non-ORE/No loss incurred & \\
\hline & 5. Defaulted & & \\
\hline Methodology & Multinomial logistic & Logistic & Regression \\
\hline
\end{tabular}

depend on whether or not the bank acquires title, we analyze the severity of loss in stages. We first predict the probability that the title of the loan is acquired through foreclosure, i.e., the loan is transferred to owned real estate (ORE). We then predict the probability that a loss is incurred on the loan. This categorization was found to provide better predictions of loss, and it is consistent with the financial institution's internal reporting and monitoring procedures.

The final component models the severity of the loss. We first predict the probability that the remaining balance is totally lost. For loans which incur partial losses, we then model the proportion of the outstanding balance lost.

The output of the forecasting model describes many aspects of model behavior, both on a yearly basis, a five year interval, and over the remaining life of the portfolio (30 years). We will focus on four performance measures in this paper which reflect the lifetime performance of the portfolio:

- Adjusted Severity (ADJSEV): average loss incurred on defaulted loans with non-zero losses;

- Percent Default (PCTDFLT): expected percent of loans defaulted;

- Percent Loss (PCTLOSS): expected percent of current net receivables lost;

- Time on books (ONBOOKS): expected length of time the loans in the portfolio remain active.

The models are refit on a quarterly basis to allow for seasonal patterns in delinquency rates, as well as changes in portfolio composition as active loans go off the books, new loans are initiated, and blocks of loans are purchased from or sold to other lenders.
Markovian models of mortgage performance have been proposed as alternatives to discrete event simulation in evaluating mortgage-backed securities, although the modeling tradeoff between simplicity and realism is acknowledged (Zipkin 1993). This tradeoff is important for our application since the modeling and analysis is not a one-time project, but is conducted quarterly basis after refitting the component models. A better understanding of the trade-off will either support the use of expected values or demonstrate the need to estimate performance variability.

In this paper, we investigate three causes of performance variation: variation attributable to uncertainty about future economic conditions and loan characteristics, variation due to the use of estimated coefficients in the component models, and the inherent variation which results from individual loans' discrete state transitions rather than expected value performances. In Section 2, we show how elliptical experimental designs can be used in a tolerance analysis framework to model performance variability for new loans in the portfolio. In Section 3, we present results from a highly fractionated factorial experiment to assess the model's sensitivity to the estimated coefficients of the component submodels. We run the model in a discrete event simulation mode in Section 4 to provide bounds on the mean performance measures computed from the base model. Our conclusions are provided in Section 5.

\section{TOLERANCE EXPERIMENT: SCE- NARIO ANALYSIS}

Throughout this paper, we concentrate on one small segment (6508 loans) of the portfolio: fixed-rate uninsured loans in southern California with an initial loan balance of $\$ 150,000$ or less. A single run of the fore- 
Table 2: Coded Factor Levels for Scenario Analysis

\begin{tabular}{ccccccc} 
DELUNEM & MKTAPPR & CONVRATE & LTV & AMOUNT & INTRATE & TERM \\
\hline-0.692 & -0.692 & -1 & +1.344 & +1.344 & +1 & -1 \\
+1.233 & -1.233 & -1 & -1.344 & -1.344 & +1 & +1 \\
-1.233 & +1.233 & -1 & -0.439 & -0.439 & -1 & +1 \\
+0.692 & +0.692 & -1 & +0.439 & +0.439 & -1 & -1 \\
-0.692 & -0.692 & +1 & +0.439 & +0.439 & -1 & +1 \\
+1.233 & -1.233 & +1 & -0.439 & -0.439 & -1 & -1 \\
-1.233 & +1.233 & +1 & -1.344 & -1.344 & +1 & -1 \\
+0.692 & +0.692 & +1 & +1.344 & +1.344 & +1 & +1 \\
\hline
\end{tabular}

casting model for this segment takes just under four minutes of CPU time on a Sparc 20 with four coprocessors at the University of Missouri-St. Louis, and substantially longer on the financial institution's older, IBM mainframe. Previously, scenario analysis efforts were performed in an ad hoc manner because of the time involved. Full portfolios may contain over 90,000 loans.

\subsection{Initial Experiment}

Monte Carlo sampling for scenario analysis is a timeconsuming activity. It is complicated by the facts that the economic variables are correlated, both with each other and over time, and the coefficients in the component models are related to each other. Instead, we adapt the so-called tolerance analysis of Taguchi $(1986,1987)$ by using response surface metamodeling methods to examine the robustness of the comprehensive forecasting model.

Response surface metamodels are efficient scenario analysis tools. Once constructed, they permit an analyst to examine many alternatives without re-running the simulation. In the tolerance analysis context, response surface metamodels can be used to assess the system's overall performance, to attribute the overall performance variation to variation in the factors, and to determine whether or not changes in the variability of the factors would increase or decrease the performance variability (Myers et al. 1992; Sanchez et al. 1994a, 1996).

We consider seven factors in our experiment. The first three represent sources of variation in the general economic environment, and are not controllable by the financial institution, and so can be referred to as external noise factors. The latter four are internal sources of noise in that they represent characteristics which vary from loan to loan across the portfolio. These factors can be influenced by lending policies for new loans. For example, new loans with low down- payments (high LTVs) may be charged higher interest rates. Regardless, the level to which the institution segments the portfolio for projection purposes will influence the noise factors' variability. The factors are:

- DELUNEM: A constant yearly percent change in unemployment rate for 1-4 years in the future (for 5 years and out, this was held at zero);

- MKTAPPR: An index value representing a constant yearly change in market appreciation rates for 1-4 years in the future (for 5 years and out, this was held at its average value);

- CONVRATE: The conventional interest rate on new 30 year fixed-rate mortgages;

- LTV: The loan-to-value ratio at origination;

- AMOUNT: The loan amount;

- INTRATE: The interest rate on the loan;

- TERM: The term of the loan.

Two sets of jointly correlated factors exist. The first set is DELUNEM and MKTAPPR. These environmental factors vary by year and by principal metropolitan statistical area, but had a correlation of -.521 over the prior five year history when DELUNEM was lagged by one year. In other words, increases in local unemployment rates led to subsequent decreases in market appreciation. LTV and AMOUNT constitute the second set of correlated factors. Since we are interested in predicting the performance for new loans, and inflation makes comparisons of dollar amounts questionable over long periods of time, we calculated the correlation for only the newest loans (with a vintage of one year or less.) These had a strong positive correlation of .807. Higher loan-tovalue ratios tended to be associated with larger loans, perhaps indicating more difficulty in raising money for the down payment. 
Table 3: Transmitted Variances as Percentages of Total Performance Measure Variances

\begin{tabular}{|c|c|c|c|c|}
\hline \multirow{2}{*}{$\begin{array}{l}\text { Factor } \\
\text { Groupings }\end{array}$} & \multicolumn{4}{|c|}{ Performance Measure } \\
\hline & ADJSEV & PCTDFLT & PCTLOSS & ONBOOKS \\
\hline DELUNEM & $0.00 \%$ & $2.56 \%$ & $6.67 \%$ & $2.23 \%$ \\
\hline MKTAPPR & $0.07 \%$ & $3.25 \%$ & $5.47 \%$ & $1.20 \%$ \\
\hline Cross term & $-0.01 \%$ & $-3.03 \%$ & $-6.55 \%$ & $-1.72 \%$ \\
\hline Net Effect & $0.06 \%$ & $2.78 \%$ & $5.59 \%$ & $1.72 \%$ \\
\hline CONVRATE & $0.00 \%$ & $0.01 \%$ & $.02 \%$ & $.11 \%$ \\
\hline LTV & $0.18 \%$ & $44.52 \%$ & $26.20 \%$ & $87.26 \%$ \\
\hline AMOUNT & $92.77 \%$ & $5.35 \%$ & $11.08 \%$ & $2.58 \%$ \\
\hline Cross term & $6.65 \%$ & $24.91 \%$ & $27.50 \%$ & $-24.23 \%$ \\
\hline Net Effect & $99.60 \%$ & $74.78 \%$ & $64.78 \%$ & $65.61 \%$ \\
\hline INTRATE & $.09 \%$ & $21.16 \%$ & $27.40 \%$ & $12.28 \%$ \\
\hline TERM & $.25 \%$ & $1.27 \%$ & $2.21 \%$ & $20.28 \%$ \\
\hline Total & $100.00 \%$ & $100.00 \%$ & $100.00 \%$ & $100.00 \%$ \\
\hline
\end{tabular}

With only seven factors, we can construct a saturated two-level factorial experiment. The design matrix (in natural units) is shown in Table 2. The levels for the non-correlated factors (CONVRATE, INTRATE, and TERM) were set at approximately one standard deviation above and below the mean. (The levels for TERM were rounded slightly since most mortgages have terms in multiples of 12 months.) These levels are recommended for discrete sampling of a normal distribution, since they result in a twopoint distribution with the same mean and standard deviation as the underlying distribution.

For the correlated factor pairs, the factor levels were calculated using the experimental designs of Sanchez (1994b). Design points are located at the major and minor axes of an ellipse, rather than at corners or faces of the cubes typically used to graphically represent factorial designs. Let $\mu_{i}$ and $\sigma_{i}$ denote the mean and standard deviation of factor $i(i=1,2)$. The ellipse's shape is a function of the correlation $\rho$ between the two factors:

$$
\begin{array}{cl}
\text { Major axes: } & \left(\mu_{1}+\sigma_{1} \sqrt{1+\rho}, \mu_{2}+\sigma_{2} \sqrt{1+\rho}\right) \\
& \left(\mu_{1}-\sigma_{1} \sqrt{1+\rho}, \mu_{2}-\sigma_{2} \sqrt{1+\rho}\right) \\
\text { Minor axes: } \quad & \left(\mu_{1}-\sigma_{1} \sqrt{1-\rho}, \mu_{2}+\sigma_{2} \sqrt{1-\rho}\right) \\
& \left(\mu_{1}+\sigma_{1} \sqrt{1-\rho}, \mu_{2}-\sigma_{2} \sqrt{1-\rho}\right)
\end{array}
$$

Means and standard deviations can be estimated from historical data or expert opinion.

After running the experiment, we can construct models for performance measure $P M_{j}$ as functions of the noise factors $W_{h}(h=1, \ldots, w)$ :

$$
P M_{j} \approx \hat{\beta}_{0, j}+\sum_{h=1}^{w} \hat{\beta}_{h}, j W_{h}
$$

Treating the regression coefficients in equation (1) as constants, we obtain

$$
\sigma_{j}^{2} \approx \sum_{h=1}^{w} \hat{\beta}_{h, j}^{2} \sigma_{h}^{2}+2 \sum_{h=1}^{w} \sum_{k=h+1}^{w} \rho_{h, k} \hat{\beta}_{h, j} \hat{\beta}_{k, j} \sigma_{h} \sigma_{k} .
$$

Equation (2) estimates the overall variation in performance measure $j$. For a noise factor $h$ which is uncorrelated with all other noise factors, its contribution to this overall variance is called the transmitted variance and is equal to $\hat{\beta}_{h, j}^{2} \sigma_{h}^{2}$. For correlated factor pairs, the transmitted variance cannot be separated completely. For example, if factors 1 and 2 are correlated then their joint transmitted variance to performance measure $j$ is equal to

$$
\hat{\beta}_{1, j}^{2} \sigma_{1}^{2}+\hat{\beta}_{2, j}^{2} \sigma_{2}^{2}+2 \rho_{1,2} \hat{\beta}_{1, j} \hat{\beta}_{2, j} \sigma_{1} \sigma_{2} .
$$

Noise factor assessment refers to the attribution of variance in a performance measure to known sources of variation. The noise factor assessments for all four performance measures are provided in Table 3. (Although the transmitted variances are approximate, percentages are shown to two decimal places to facilitate reassessment as in Section 3.2.) For this experiment, the net transmitted variance from the correlated loan characteristics (AMOUNT and LTV) dominates for all four performance measures. This is not surprising for ADJSEV: one would expect the magnitude of the loss to depend on the size of the loan. LTV 
appears to be the most influential of the pair for predicting PCTDFLT and ONBOOKS. INTRATE impacts the overall loss, but through the transition and loss behavior rather than through the severity component. External market conditions had surprisingly little impact on the overall performance, and loan term was only a factor for predicting the expected time on the books.

Note that a traditional factorial design and analysis would be inappropriate for two reasons. First, certain combinations of the factors are not likely to occur, thus the goal of obtaining the same variability in the sampling distribution as in the underlying distribution would not be realized by setting all factor levels at $\mu \pm \sigma$. Second, the original forecasting model (metamodel) was not constructed using similar combinations. The results would be highly suspect for such dramatic extrapolations. The analysis differs as well. For example, without considering the high positive correlation between LTV and AMOUNT, one would underestimate the overall variance in PCTDFLT and PCTLOSS by over $24 \%$. Conversely, the variance of ONBOOKS would be overestimated if the correlation of LTV and AMOUNT were ignored.

\subsection{Model use and refinement}

Metamodels can often be simplified by removing statistically insignificant terms. Normal probability plots are useful for this activity if few or no degrees of freedom are available for error estimation, as in this experiment. However, we advise caution in oversimplification when two or more noise factors are correlated. The sampling scheme is not orthogonal, so if $|\rho|$ is near one, then the standard errors of the correlated regression coefficients may be inflated due to multicollinearity. The effect of correlation on the overall variance is computed through the metamodel coeffcients, so excluding a marginally insignificant main effect from the model may have noticeable impacts on system evaluation and noise factor assessment due to the exclusion of interaction terms. Thus, we elect to retain both terms of a correlated factor pair in the metamodel even if only one is significant. We used a cutoff of $\alpha=.20$ because of low degrees of freedom and our desire to achieve models with accurate fits. For the 8-run tolerance experiment, reduced metamodels with relatively high $R^{2}$ values were constructed for each of the four performance measures:

- ADJSEV: $R^{2}=.999$ with LTV, AMOUNT and TERM.

\footnotetext{
- PCTDFLT: $R^{2}=.941$ with LTV, AMOUNT and INTRATE.
}

- PCTLOSS: $R^{2}=.889$ with LTV, AMOUNT and INTRATE.

- ONBOOKS: $R^{2}=.982$ with LTV, AMOUNT, CONVRATE, INTRATE and TERM.

If the reduced metamodels are used for noise factor assessment, the transmitted variance percentages are zero for factors excluded from the metamodels, and not materially different from those reported in Table 3 for the factors retained.

Note that statistical significance in the metamodels does not necessarily imply practical importance. Since the transmitted variances depend on the component variances (and covariances), a term may be statistically significant in the metamodel yet correspond to a factor with transmitted variance near zero. For example, TERM is statistically significant in the model of ADJSEV, but accounts for only $0.25 \%$ of the variability of this performance measure.

One benefit of tolerance analysis is the ease of modifying the results to predict the effects of changes in the underlying sources of noise. For example, the financial institution might be interested in forecasting the future loss percentage for a smaller portfolio segment: new loans with half the standard deviations of loan-to-value and amount. The transmitted variance of this factor grouping would be reduced by a factor of four. After re-standardizing, the net effect of DELUNEM and MKTAPPR would account for $10.87 \%$ of the variance in PCTLOSS; the net effect of LTV and AMOUNT would account for $31.50 \%$, the INTRATE would account for $53.30 \%$ and the TERM would account for $4.29 \%$. (CONVRATE's contribution remains negligible at $0.04 \%$.) Similarly, if the volatility in market conditions increased, one could re-estimate the transmitted variances to determine the impacts (if any) on performance. Such transmitted variance reassessments require no additional computer runs and may help guide scenario analysis efforts. For example, from Table 3 we see that rerunning the base model with different conventional rates will have no appreciable effect on any of the four performance measures if the conventional rate ranges from $7.45 \%$ to $8.45 \%$. Even doubling the range would increase performance variability by at most $0.33 \%$, according to our metamodels. However, it is important to remember that the metamodels are only approximations of the true response surface. If large changes in noise factor variance are anticipated, a confirmation run using new factor settings should be made. If the confirmation run's performance measures are not close to those predicted, the new information can be used to develop into more appropriate metamodels of performance. 
Table 4: Transmitted Variance Percentages: Coefficient Sensitivity Experiment

\begin{tabular}{lcrrrr} 
& & \multicolumn{4}{c}{ Performance Measure } \\
\cline { 3 - 6 } $\begin{array}{l}\text { Coefficient } \\
\text { Grouping }\end{array}$ & $\begin{array}{c}\text { \# Coeffs. } \\
\text { Perturbed }\end{array}$ & ADJSEV & PCTDFLT & PCTLOSS & ONBOOKS \\
\hline $\begin{array}{l}\text { Transition } \\
\quad \text { from Current }\end{array}$ & 32 & $57 \%$ & $86 \%$ & $55 \%$ & $66 \%$ \\
$\begin{array}{l}\text { Transition } \\
\quad \text { from Del. 30-89 }\end{array}$ & 8 & $2 \%$ & $0 \%$ & $0 \%$ & $0 \%$ \\
$\begin{array}{l}\text { Transition } \\
\quad \text { from Del. 90+ }\end{array}$ & 8 & $15 \%$ & $14 \%$ & $5 \%$ & $32 \%$ \\
$\begin{array}{l}\text { Loss } \\
\text { Severity }\end{array}$ & 11 & $27 \%$ & $0 \%$ & $40 \%$ & $1 \%$ \\
\hline Total \%: & 2 & $0 \%$ & $0 \%$ & $0 \%$ & $0 \%$ \\
\hline
\end{tabular}

\subsection{Validation Experiment}

A common concern when using first-order metamodels is that they may not adequately approximate the response surface. To validate the results of Section 3.1 and 3.2 we performed a 64-run half-fraction experiment, which allows us to estimate all main effects and two-way interactions. The larger number of data points provides ample degrees of freedom for assessing statistical significance, although the p-values for positively correlated factor pairs will still be inflated. Both main-effect models and second-order models were examined. For ADJSEV, the reduced model contained the same three terms as the 8-run metamodel: no interaction effects were present. MKTAPPR was significant for the other three performance characteristics, and CONVRATE was significant for PCTLOSS and ONBOOKS. The effects due to variation in economic conditions were thus better identified in the 64-run experiment than in the 8-run experiment. Few interactions appeared to influence performance. Only three are significant using $\alpha=.05$ (LTV by MKTAPPR, LTV by AMOUNT, and LTV by INTRATE, all in the metamodel for PCTDFLT). One more was present at $\alpha=.10$ (LTV by AMOUNT in the metamodel for ONBOOKS).

The transmitted variances are small for the factors not found to be statistically significant in the 8-run experiment, so leaving them in the model has little impact on the noise factor assessment for the present portfolio. However, statistical significance is difficult to determine with so few degrees of freedom available for error estimation, and the full model provides more accurate results if the economic forecasts change or if the portfolio is segmented differently. The latter is of interest for evaluating changes to current lending policies. In summary, it appears that the 8-run metamodels suffice for noise factor assessment of our mortgage portfolio example if all factors are retained in the metamodels and transmitted variances are estimated using equation (3).

\section{TOLERANCE EXPERIMENT: MODEL SENSITIVITY}

Recall from Table 1 that the comprehensive model makes use of fitted values from multinomial logistic, logistic, and regression analysis. For the 6508 loan segment we analyze, a total of 61 coefficients are used in the various model components. (Another 85 coefficients are needed to run the forecasting model for the full portfolio.) These coefficients are themselves estimated from previous data, so the sensitivity of the responses to errors in their estimation (or changes in the relationships over time) is of interest. However, since the model coefficients are updated quarterly to reflect more recent loan histories and environmental information, we prefer a method which is easily automated, even at the expense of some accuracy.

To this end, we modified the base forecasting program so that the coefficients could be perturbed using a nearly saturated two-level fractional factorial design with 64 runs. Each of the model coefficients is coded as $c_{i}+f_{i} s_{i}$ where $c_{i}$ and $s_{i}$ are, respectively, the mean and standard deviation of the $i$ th coefficient and $f_{i}$ is the coded factor level for the run. (For the base model calculations, all $f_{i}$ can be set to zero.) A shell script loops through all 64 design points and iteratively calls the SAS forecasting program. Note that this design treats the coefficients as independent factors although they are correlated. Because of the nature of the multinomial logistic structure, we expect the results from the tolerance experiment to be 
conservative (i.e., show wider variation) relative to results that would be achieved by a method which made use of the multivariate correlation structures.

The market conditions (unemployment, market appreciation, and conventional interest rate) were fixed at the nominal (center point) levels of the 8-run tolerance design experiment. This left four factors: original loan-to-value, loan amount, interest rate, and term. For efficiency purposes, we chose to run the experiment using the four factor level combinations of the scenario tolerance experiment augmented by a center point, rather than using the full portfolio.

The results are summarized in Table 4 . The coeffcients for the 'transitions from current' state appear to be the major source of variation for all four performance measures, with transmitted variance percentages ranging from $55 \%$ to $86 \%$ of the totals. This represents the majority of the transitions which occur over the life of loans in the portfolio. Coefficients from the 'transitions from $90+$ days delinquent' component account for $15 \%$ of the transmitted variance for ADJSEV, $14 \%$ for PCTDFLT, and $5 \%$ for PCTLOSS. Since the majority of loans entering default first pass through this late delinquency stage, the impact on the overall model is not surprising. The severity coefficients appear to impact both ADJSEV and PCTLOSS, but have little effect on the PCTDFLT or ONBOOKS. The transmitted variance of the severity coefficients is negligible relative to the other model components.

The transmitted variance percentages in Table 4 are based on full regression models. For comparison purposes, stepwise regression was used to obtain simpler models. The numbers of terms in the reduced metamodels dropped from 61 to 38, 30, 27 and 36 for ADJSEV, PCTDFLT, PCTLOSS, and ONBOOKS, respectively, but the transmitted variance percentages did not materially change.

\section{INHERENT VARIANCE ESTIMATION}

Recall that the base program relies on expected values rather than discrete event simulation to reduce the computational effort. We wished to estimate the variability of the means to determine whether or not reliance on expected behavior was reasonable. To accomplish this task, we modified the forecasting program to run as a discrete event simulation. For example, in the base program we calculate probabilities for each of the five states one year out, two years out, and so forth up to 30 years out. For the discreteevent version, we calculate the state probabilities on an individual loan basis to create multinomial dis- tributions for the the loan state after one year ha: elapsed. SAS's internal random numbers routines are then used to determine which multinomial outcome is realized. Similarly, loss transitions are randomly determined with probabilities calculated from the comprehensive forecasting model. If the loan does not enter a terminal state at the beginning of year $t$, then transition probabilities for the year $t+1$ are recalculated after accounting for the amortization.

We provide estimates of the performance measures' variability obtained using discrete event simulation in Table 5. Three different portfolios are used as the basis for simulation: (1) 1,000 replications of the center point loan from Section 3 . (2) 200 replications for each of the five loans used in the sensitivity analysis experiment of Section 3, and (3) 16 replications of the 6508 loan portfolio. In all cases, standard deviations are normalized to represent the standard error of the mean for 6508 loans.

Table 5: Standard Error Estimates for 6508 Loan Portfolio

\begin{tabular}{|c|c|c|c|}
\hline \multirow[b]{2}{*}{$\begin{array}{l}\text { Performance } \\
\text { Measure }\end{array}$} & \multicolumn{3}{|c|}{ Simulated Portfolio } \\
\hline & $\begin{array}{c}\text { Center Pt. } \\
\text { Loan }\end{array}$ & $\begin{array}{c}5 \text { Design } \\
\text { Pt. Loans }\end{array}$ & $\begin{array}{c}6508 \\
\text { Loans } \\
\end{array}$ \\
\hline ADJSEV & 60.60 & 141.06 & 518.35 \\
\hline PCTDFLT & 0.234 & 0.669 & 0.327 \\
\hline PCTLOSS & 0.007 & 0.065 & 0.058 \\
\hline ONBOOKS & 0.045 & 0.101 & 0.056 \\
\hline
\end{tabular}

We anticipated that the standard deviations computed by discrete event simulation of very small portfolios (the center point loan, or the five design point loans) would be conservative estimates of the standard deviations resulting from the entire 6508 loan simulation, in part because the larger portfolio has loans closer to maturity and thus fewer potential paths through their remaining life. If so, and if the resulting bounds were sufficiently narrow, then future estimation of the inherent variability could be accomplished efficiently using a reduced portfolio. Unfortunately, the standard deviation of ADJSEV was not approximated closely by either the center point simulation or the five design point simulation. The standard deviations estimated from the five design points are conservative for the other three performance measures. Discrete-event simulation of the center point loan underestimated the standard deviations for all four performance measures, so for our example it would not provide adequate bounds on the expected performance variability. 


\section{CONCLUDING REMARKS}

We have examined three causes of variation for four performance measures in the base forecasting model: variation due to uncertainties about future economic conditions and new loan characteristics, variation due to the use of estimated coefficients for component models, and inherent variation due to the stochastic nature of loan behavior within the portfolio. The central limit theorem means the inherent variation is less a concern for large portfolios. Similarly, if the coefficients in the component models are estimated from a large number of loans, their standard errors will tend to be smaller and their effect will be dampened. However, the noise factor assessments from tolerance analysis do not directly depend on the size of the portfolio for which scenario analysis is conducted.

Taken together, these three causes of variation can be used to provide bounds around the expected performance measures computed from the base forecasting model. When this is done for the five design point loans, the overall performance variance computed during tolerance analysis was the major source of uncertainty for ADJSEV, the inherent variance was the major source of difference for PCTDFLT and PCTLOSS, and the uncertainty concerning the coefficients had the major impact on ONBOOKS. This indicates that all types of variation should be considered in order to obtain bounds on the expected portfolio performance.

We have investigated methods for efficient response variability assessment. Small experimental designs were be used to augment or replace extensive discrete event simulation to predict the performance of home mortgage portfolios. We found that strong correlations between factors must be considered when designing and analyzing these experiments in order to obtain accurate noise factor assessments. In addition to one-time insights into a particular portfolio segment's behavior, these methods hold promise for adaptation to an ongoing process of model refitting and revision.

\section{REFERENCES}

Myers, R. H., A. I. Khuri and G. Vining. 1992. Response surface alternatives to the Taguchi robust parameter design approach. The American Statistician 46(2): 131-139.

Sanchez, S. M. 1994a. Experiment designs for system assessment and improvement when noise factors are correlated. In Proceedings of the 1994 Winter Simulation Conference, ed. J. D. Tew, M. S. Manivannan, D. A. Sadowski and A. F. Seila, 290-296. In- stitute of Electrical and Electronic Engineers, Orlando, Florida.

Sanchez, S. M. 1994b. A robust design tutorial. In Proceedings of the 1994 Winter Simulation Conference, ed. J. D. Tew, M. S. Manivannan, D. A. Sadowski and A. F. Seila, 106-113. Institute of Electrical and Electronic Engineers, Orlando, Florida.

Sanchez, S. M., P. J. Sanchez and J. S. Ramberg. 1996. A simulation framework for robust system design. In Concurrent design of products, manufacturing processes and systems, ed. B. Wang. New York: Gordon and Breach, forthcoming.

Smith, L. D., S. M. Sanchez and E. C. Lawrence. 1996. A comprehensive model for managing credit risk and forecasting losses on home mortgage portfolios. Decision Sciences, forthcoming.

Taguchi, G. 1986. Introduction to quality engineering, White Plains, New York: UNIPUB/Krauss International.

Taguchi, G. 1987. System of Experimental Design, Vols. 1 and 2. White Plains, New York: UNIPUB/Krauss International.

Zipkin, P. 1993. Mortgages and Markov chains: a simplified valuation model. Management Science 39: $2-16$.

\section{AUTHOR BIOGRAPHIES}

SUSAN M. SANCHEZ is Associate Professor of Management Science and Information Systems at the University of Missouri-St. Louis. Her research interests include the design and analysis of simulation experiments, selection, and data-intensive statistical methods in business and manufacturing. She is an associate editor for Operations Research and Naval Research Logistics.

L. DOUGLAS SMITH is Professor of Management Science and Information Systems at the University of Missouri-St. Louis. He holds a Ph.D. in Management Sciences from the University of Minnesota and an M.B.A. and B.Sc. (Physics) from McMaster University. Smith is a former Associate Editor of Decision Sciences. His research has emphasized the development of models and computer-based systems for the solution of managerial problems.

EDWARD C. LAWRENCE is Professor of Finance in the School of Business Administration at the University of Missouri-St. Louis. He has written extensively in the areas of banking and corporate finance. He received his Ph.D. in Finance from the Pennsylvania State University. 